\title{
Reversine inhibits proliferation, invasion and migration and induces cell apoptosis in gastric cancer cells by downregulating TTK
}

\author{
PENGFEI XIA, JIN LIANG, DI JIN and ZHANYONG JIN \\ Department of Liver-Gallbladder and Gastric Diseases, \\ Wuhan Hospital of Traditional Chinese Medicine, Wuhan, Hubei 430061, P.R. China
}

Received October 29, 2020; Accepted March 2, 2021

DOI: $10.3892 /$ etm.2021.10361

\begin{abstract}
Reversine (Rev) has been used for the treatment of a number of cancers. However, there have been no previous reports for the use of Rev for gastric cancer (GC). The aim of the present study was to investigate the effect of Rev on cell proliferation, migration, invasion and cell apoptosis in human GC cells and TTK expression. Cell Counting Kit- 8 and colony formation were used to assess cell proliferation. Wound healing and Transwell assays were performed to examine cell migration and invasion, respectively. Cell apoptosis was measured using TUNEL staining and western blotting. Reverse transcription-quantitative PCR and western blotting were performed to determine TTK expression in AGS and NCI-N87 GC cells. Rev treatment inhibited the viability of the two GC cells lines in a dose-dependent manner and suppressed their capacities of clone formation, migration and invasion. Rev-treated cells exhibited reduced matrix metalloproteinase (MMP)2/9 expression and increased apoptosis compared with those in control cells. In addition, expression of the anti-apoptotic protein Bcl-2 was significantly decreased, whilst the expression levels of the pro-apoptotic factors Bax and cleaved-caspase-3/9 were increased by Rev treatment compared with that in the control group that were not treated with Rev. In addition, TTK protein expression was decreased in cells treated with Rev compared with that in untreated cells. However, overexpression of TTK significantly reversed the aforementioned effects of Rev in GC cells. These results suggest that Rev may inhibit the proliferation, invasion and migration of GC cells whilst inducing cell apoptosis by suppressing TTK expression. Therefore, Rev may confer potential properties as a therapeutic anti-cancer agent. Additionally, TTK may serve as a molecular target for the treatment of gastric cancer.
\end{abstract}

Correspondence to: Dr Zhanyong Jin or Dr Di Jin, Department of Liver-Gallbladder and Gastric Diseases, Wuhan Hospital of Traditional Chinese Medicine, 303 Sixin Avenue, Wuhan, Hubei 430061, P.R. China

E-mail: jinzhanyong@yeah.net

E-mail: jingdijd@yeah.net

Key words: gastric cancer, reversine, TTK, migration, apoptosis

\section{Introduction}

Gastric cancer (GC) is one of the major causes of cancer-related mortality and is a major health burden worldwide (1). Each year there are $\sim 1$ million new cases of GC worldwide, prompting the World Health Organization to declare it a public health concern (2). Patients with GC typically exhibit the 'three high and three low' characteristic, whereby the incidence, metastasis and mortality rates are high whereas the early diagnosis rate, radical resection rate and 5-year survival rate are low (3). As surgical techniques improve and progress is made in traditional radiotherapy, chemotherapy and the implementation of neoadjuvant therapy, the 5-year survival rate for early GC can reach $>95 \%$ (2). However, the low rate of early diagnosis $(>70 \%)$ means that most patients will develop advanced-stage disease, resulting in the optimal surgical window being missed, worsening the overall prognosis (2). At present, the main treatment strategy for advanced GC is the combination of neoadjuvant chemoradiotherapy, molecular-targeted therapy and immunotherapy (2). However, development of novel effective therapeutic agents or the discovery of novel therapeutic targets for GC treatment remains urgently sort after.

Reversine (Rev) is a 2,6-diamino-substituted purine analogue that was originally used as a depolarizer to control cellular dedifferentiation and may ultimately prove to be useful for in vivo stem cell biology and therapy (4). Rev induces mitotic catastrophe, cell cycle arrest, polyploidy and cell apoptosis in a number of human cancer cell types, including in non-small cell lung cancer and breast cancer (5-7). A previous study has shown that Rev treatment resulted in cytotoxicity in human colorectal cancer (CRC) cells and inhibited cell migration by modulating the JNK signaling pathway (8). Additionally, Rev treatment suppressed tumor progression by inhibiting cell proliferation, inducing apoptosis and cell cycle arrest through upregulation of the Fas and death receptor 5 signaling pathways in CRC cells (9). However, there have been no previous reports on the effect of Rev on GC cells.

The protein kinase TTK, which is also known as monopolar spindle 1 or Mps1, has been documented to serve critical roles in malignant diseases, including hepatocellular carcinoma, breast cancer, glioblastoma and pancreatic cancer, where has been reported to promote cell proliferation, invasion and 
epithelial-to-mesenchymal transition (10-13). Frameshift mutations of TTK may alter cell cycle control in the affected cells and contribute to pathogenesis of GC and CRC with high microsatellite instability (14). Lower expression levels of TTK was also reported to be associated with superior prognosis of patients with glioblastoma and breast cancer $(13,15)$. In GC, TTK may contribute to tumorigenesis (16), such that TTK expression was found to be higher in the six GC cell lines AGS, MKN-45, SGC 7901, KATO III, N-87 and SNU-1 tested compared with that in the normal gastric cell line GES-1, suggesting that TTK may be a new therapeutic target for GC (17). However, the role of TTK and the relationship between TTK and Rev in the regulation of GC physiology remain ambiguous.

Therefore, the aim of the present study was to investigate the effect of Rev on GC and its association with TTK in human GC cells.

\section{Materials and methods}

Cell culture and reagents. The two human GC cell lines AGS and NCI-N87, in addition to the human immortalized gastric epithelial cell line (GES-1) were obtained from the American Type Culture Collection. GC cells were cultured in RPMI-1640 medium supplemented with 10\% FBS (Gibco; Thermo Fisher Scientific, Inc.) and penicillin-streptomycin $(100 \mathrm{U} / \mathrm{ml})$ at $37^{\circ} \mathrm{C}$ with $5 \% \mathrm{CO}_{2}$. By contrast, GES-1 cells were grown in DMEM (Gibco; Thermo Fisher Scientific, Inc.) containing $10 \% \mathrm{FBS}$ with $100 \mathrm{U} / \mathrm{ml} \mathrm{P} / \mathrm{S}$ at $37^{\circ} \mathrm{C}$ with $5 \% \mathrm{CO}_{2}$. Reversine was purchased from Cayman Chemical Company and was kept as a $10 \mathrm{mM}$ solution in DMSO.

Cell viability assay. Cell viability was detected by Cell Counting Kit-8 (CCK-8) assay (Dojindo Molecular Technologies, Inc.). AGS and NCI-N87 cells were seeded into a 96-well plate at a density of $1 \times 10^{4}$ cells/well before they were incubated for $24 \mathrm{~h}$ at $37^{\circ} \mathrm{C}$. The cells were then treated with different concentrations of $\operatorname{Rev}(0,0.5,1,5,10$ and $20 \mu \mathrm{M})$ with DMSO (Sigma-Aldrich; Merck KGaA) for 24 and $48 \mathrm{~h}$ at $37^{\circ} \mathrm{C}$. Subsequently, the cells were treated with $10 \mu \mathrm{l} \mathrm{CCK}-8$ solution (Dojindo Molecular Technologies, Inc.) for $2 \mathrm{~h}$ at $37^{\circ} \mathrm{C}$. After treatment, absorbance at $450 \mathrm{~nm}$ was measured in each well using a microplate reader (Varioskan ${ }^{\circledR}$ Flash; Thermo Fisher Scientific, Inc.). Each experiment was conducted in triplicate wells and was repeated $\geq$ three times.

Transfection. AGS and NCI-N87 cells were seeded (4x10 $/$ well) into six-well plates and incubated at $37^{\circ} \mathrm{C}$ overnight. For TTK overexpression, TTK overexpression plasmid (Oe-TTK) and empty plasmid (Oe-NC) were designed and constructed by Shanghai GenePharma Co., Ltd. The cells were transfected with Oe-TTK or Oe-NC using Lipofectamine ${ }^{\circledR} 2000$ reagent (Invitrogen; Thermo Fisher Scientific, Inc.) for $6 \mathrm{~h}$. The final concentration of the plasmids was $2 \mu \mathrm{g} / \mathrm{ml}$. The overexpression of TTK expression following Oe-TTK transfection was verified by reverse transcription-quantitative PCR (RT-qPCR), after which the transfected cells were treated with $20 \mu \mathrm{M}$ Rev for $24 \mathrm{~h}$ at $37^{\circ} \mathrm{C}$.

Colony formation assay. AGS and NCI-N87 cells were seeded into six-well plates at a low density $\left(1 \times 10^{3}\right)$ in each well. Cells were then incubated for $24 \mathrm{~h}$ and then treated with $20 \mu \mathrm{M}$ Rev for $24 \mathrm{~h}$ at $37^{\circ} \mathrm{C}$. After 12 days incubation in RPMI-1640 medium with $10 \% \mathrm{FBS}$ at $37^{\circ} \mathrm{C}$, the plates were washed with PBS and stained with $0.1 \%$ crystal violet at room temperature for $5 \mathrm{~min}$. Colony formation images were captured using a camera (original magnification x100; Olympus Corporation). Colonies, defined as clusters of $>50$ cells, were counted before colony intensities were calculated using the ImageJ software Version 1.8.0 (National Institutes of Health). Each experiment was repeated $\geq$ three times.

Wound healing assay. AGS and NCI-N87 cells were seeded into a $60-\mathrm{mm}$ dish and cultured for $24 \mathrm{~h}$ at $37^{\circ} \mathrm{C}$. When the cells became confluent, the monolayer was scratched using a sterile $1 \mathrm{ml}$ pipette tip. The monolayer was then washed three times with PBS to remove cell debris and incubated in RPMI-1640 medium containing 2\% FBS. After 48-h incubation at $37^{\circ} \mathrm{C}$, images of wound healing were captured under a light microscope (magnification, x200). The migration rate was calculated based on the formula: (Wound width at $0 \mathrm{~h}$-wound width at $24 \mathrm{~h}$ )/wound width at $0 \mathrm{~h} \times 100 \%$. Each experiment was repeated $\geq$ three times.

Invasion assay. Invasion assay was performed using a Transwell chamber with $8-\mu \mathrm{m}$ pores (Corning, Inc.). The upper chamber of the Transwell was first coated with $3 \mathrm{mg} / \mathrm{ml}$ Matrigel (BD Biosciences) and incubated at $37^{\circ} \mathrm{C}$ for $1 \mathrm{~h}$. Cells were incubated in RPMI-1640 containing $1 \%$ FBS and treated with $20 \mu \mathrm{M}$ Rev for $24 \mathrm{~h}$ at $37^{\circ} \mathrm{C}$. The cells were trypsinized and suspended at a final concentration of $5 \times 10^{5}$ cells/ml in RPMI-1640 containing $1 \%$ FBS. Cell suspensions were then loaded into the upper compartment. The lower chamber was added with $600 \mu 1$ RPMI-1640 medium containing 10\% FBS. After incubation for $48 \mathrm{~h}$ at $37^{\circ} \mathrm{C}$, the cells on the surface of the upper chamber was wiped off. The invaded cells on the lower chamber were fixed with $100 \%$ methanol for $15 \mathrm{~min}$ at room temperature, stained with $0.5 \%$ crystal violet for $10 \mathrm{~min}$ at room temperature, and captured under a light microscope (magnification, x200). Five randomly chosen fields were counted for each group per chamber. Each experiment was repeated $\geq$ three times.

TdT-mediated dUTP nick-end labeling (TUNEL) assay. AGS and NCI-N87 cells were seeded (4x10 $/$ well) into six-well plates and incubated at $37^{\circ} \mathrm{C}$. After treatment with $20 \mu \mathrm{M}$ Rev in the presence and absence of Oe-TTK for $24 \mathrm{~h}$ at $37^{\circ} \mathrm{C}$, cells were fixed with $4 \%$ paraformaldehyde for $30 \mathrm{~min}$ at room temperature and permeabilized with $0.5 \%$ Triton X-100 for $10 \mathrm{~min}$ at room temperature. After washing three times with PBS, the cells were the cells were added with $50 \mu 1$ TUNEL (cat. no. 11684817910; Roche diagnostics) at $37^{\circ} \mathrm{C}$ for $1 \mathrm{~h}$. After washing with PBS, the cells were treated with $1 \mu \mathrm{g} / \mathrm{ml}$ DAPI at room temperature for 5 min. DNA fragmentation was visualized by TUNEL assay according to the manufacturer's protocols. Finally, fluorescence images were obtained using a confocal microscope (Carl Zeiss AG) at magnifications of x200. In total, five randomly chosen fields were counted for each group per chamber. Each experiment was repeated $\geq$ three times.

$R T-q P C R$. Total RNA extraction in cells was performed using TRIzol reagent (Invitrogen; Thermo Fisher Scientific, Inc.) according to the manufacturer's protocol. Reverse transcription 


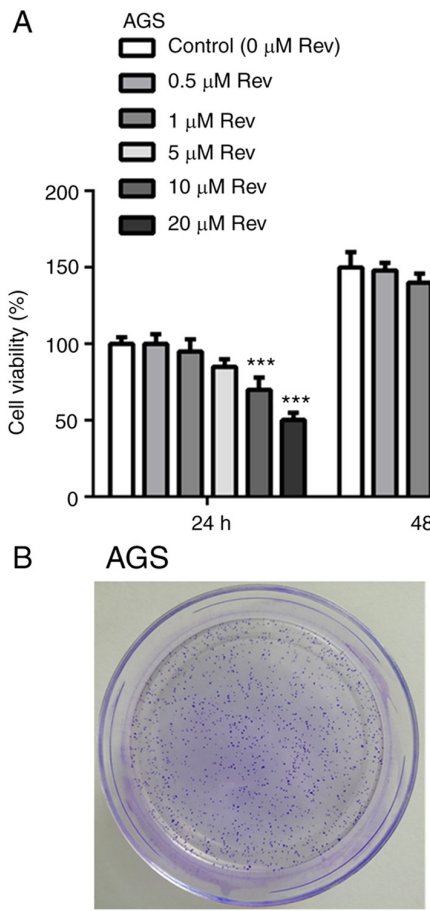

Control

$\mathrm{NCl}-\mathrm{N} 87$

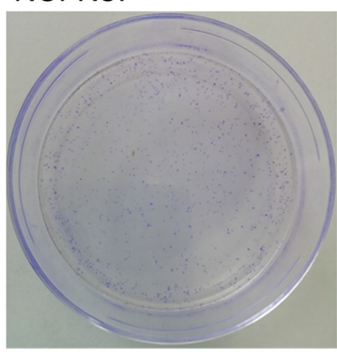

Control

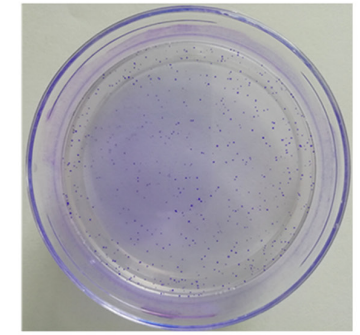

$20 \mu \mathrm{M}$ Rev

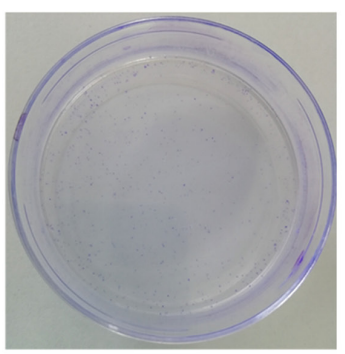

$20 \mu \mathrm{M}$ Rev
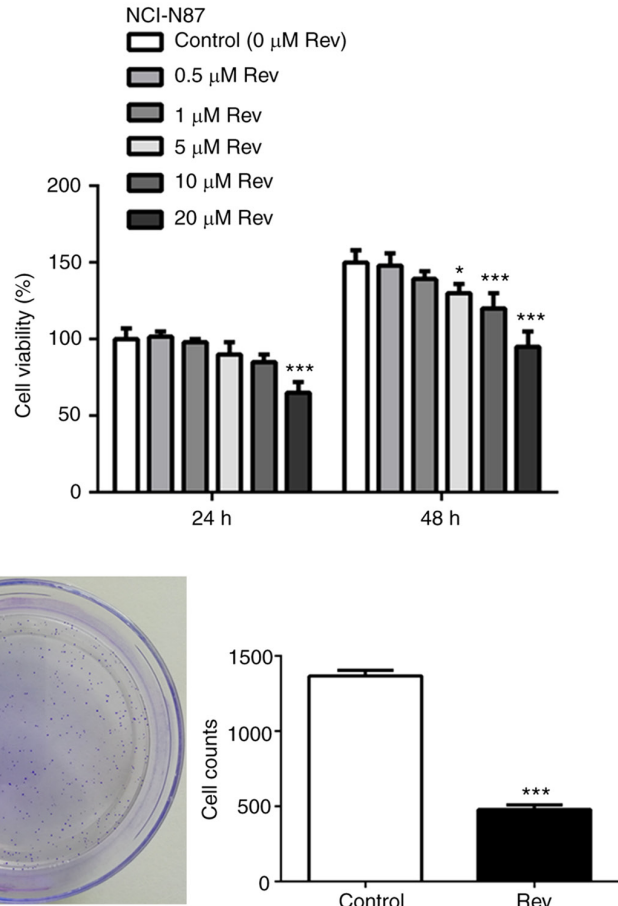

Control

Rev

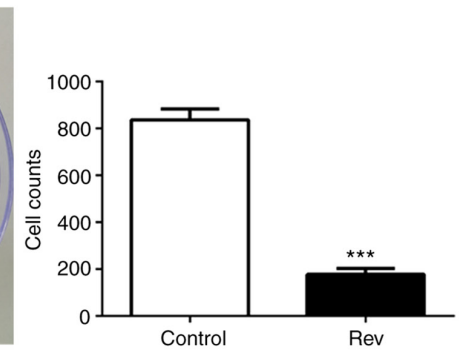

Figure 1. Reversine reduces cell viability and colony formation of human gastric cancer cells. (A) Human gastric cancer cell lines AGS and NCI-N87 were either exposed to $\operatorname{Rev}(0,0.5,1,5,10$, and $20 \mu \mathrm{M})$ for 24 and $48 \mathrm{~h}$. Subsequently, cell viability was measured using CCK-8 assay. (B) AGS and NCI-N87 cells were treated with $\operatorname{Rev}(20 \mu \mathrm{M})$ for $24 \mathrm{~h}$ before cell colony formation was observed under an invert microscope following crystal violet staining. Magnification, $\mathrm{x} 100 .{ }^{*} \mathrm{P}<0.05,{ }^{* *} \mathrm{P}<0.01$ and ${ }^{* * *} \mathrm{P}<0.001$ vs. Control. Rev, reversine.

was performed using a RevertAid First Strand cDNA Synthesis Kit (cat. no. K1622; Thermo Fisher Scientific, Inc.) and the reaction was incubated at $25^{\circ} \mathrm{C}$ for $5 \mathrm{~min}, 42^{\circ} \mathrm{C}$ for $30 \mathrm{~min}, 85^{\circ} \mathrm{C}$ for $5 \mathrm{~min}$ and then kept at $4^{\circ} \mathrm{C}$ for $5 \mathrm{~min}$. cDNA was prepared for amplification. FastStart ${ }^{\mathrm{TM}}$ Universal SYBR ${ }^{\circledR}$-Green Master Mix (Sigma-Aldrich; Merck KGaA) was used for qPCR following the manufacturer's protocol. The thermocycling conditions were as follows: Initial denaturation at $95^{\circ} \mathrm{C}$ for $5 \mathrm{~min}$, followed by 35 cycles of denaturation $\left(45 \mathrm{sec}\right.$ at $\left.95^{\circ} \mathrm{C}\right)$, annealing $\left(45 \mathrm{sec}\right.$ at $\left.60^{\circ} \mathrm{C}\right)$ and extension $\left(8 \mathrm{~min}\right.$ at $\left.68^{\circ} \mathrm{C}\right)$, before a final extension at $68^{\circ} \mathrm{C}$ for $10 \mathrm{~min}$. GAPDH was used for normalization, where gene expression was calculated using the $2^{-\Delta \Delta \mathrm{Cq}}$ method (18). qPCR amplification was performed using the following primers: TTK forward, 5'-CGCAGCTTTCTG TAGAAATGGA-3' and reverse, 5'-GAGCATCACTTAGCG GAACAC-3' and GAPDH forward, 5'-GGTGGTCTCCTC TGACTTCAACA-3' and reverse, 5'-GTTGCTGTAGCCAAA TTCGTTGT-3'. Each experiment was repeated $\geq$ three times.

Western blotting. Cell lysates were prepared in RIPA buffer (Beyotime Institute of Biotechnology) containing $1 \%$ protease and
$1 \%$ phosphatase inhibitor cocktail (Sigma Aldrich; Merck KGaA) on ice. Bicinchoninic acid method was used to measure protein concentration. Equal amounts of protein lysates (30 $\mu \mathrm{g} /$ lane) were mixed with loading buffer, separated by $10 \%$ SDS-PAGE and transferred onto PVDF membranes (Invitrogen; Thermo Fisher Scientific, Inc.). Subsequently, the membranes are blocked with $5 \%$ skimmed milk for $1 \mathrm{~h}$ at room temperature and then treated with primary antibodies (all from Abcam) against TTK (1:1,000, cat. no. ab11108), matrix metalloproteinase (MMP)-2 (1:1,000, cat. no. ab92536), MMP-9 (1:1,000, cat. no. ab38898), Bcl-2 (1:2,000, cat. no. ab182858), Bax (1:2,000, cat. no. ab32503), cleaved caspase-3 (1:500, cat. no. ab32042), cleaved caspase- 9 (1:1,500, cat. no. ab2324), caspase-3 (1:5,000, cat. no. ab32351), caspase-9 (1:1,000, cat. no. ab32539) and GAPDH (1:1,000, cat. no. ab8245) at $4^{\circ} \mathrm{C}$ overnight. The membranes were then incubated with a HRP-conjugated secondary antibody $(1: 2,000$, cat. no. 7074, Cell Signaling Technology Inc.) for $1 \mathrm{~h}$ at room temperature. The ECL ${ }^{\mathrm{TM}}$ Western Blotting Analysis System (Cytiva) and the ImageJ software (version 1.46; National Institutes of Health) were used to detect the blots. Each experiment was repeated at least three times. 
A
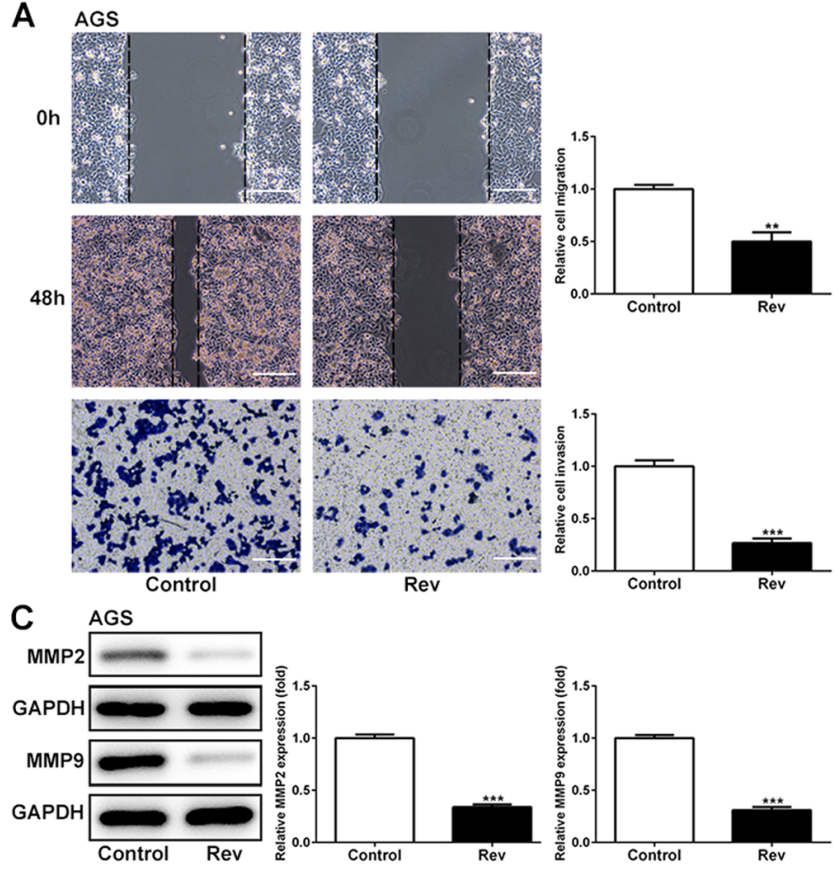
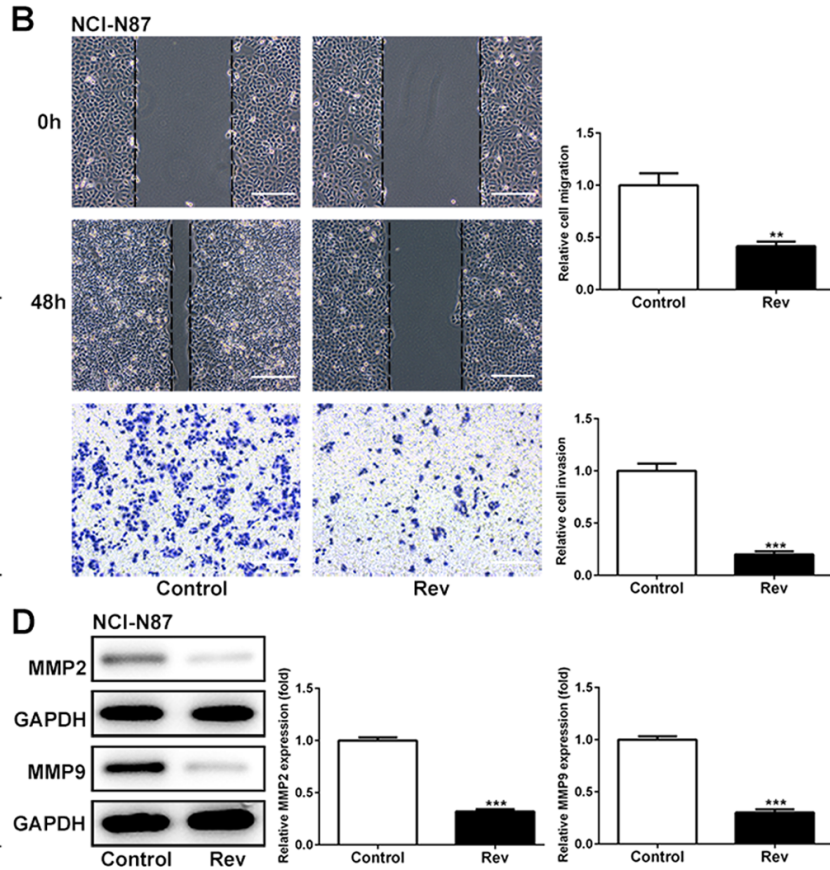

Figure 2. Reversine inhibits human gastric cancer cell migration and invasion. Wound healing and Transwell assay data in (A) AGS and (B) NCI-N87 cells. Scale bars, $40 \mu \mathrm{m}$. Representative images are shown in the left of the panel and quantitative data of migration (\%) compared with control are presented in the right panel. Western blot analysis of MMP2 and MMP9 expression in (C) AGS and (D) NCI-N87 cells, which was also quantified. ${ }^{* *}$ P $<0.01$ and ${ }^{* * *} \mathrm{P}<0.001$ vs. Control. Rev, reversine; MMP, matrix metalloproteinase.

Statistical analysis. All experiments were performed $\geq$ three times. All data were presented as the mean \pm SD. Statistical analyzes were performed using GraphPad 6 Software (GraphPad Software, Inc.). Two groups were compared using an unpaired, two-tailed Student's t-test. Multiple group comparisons were analyzed with one-way ANOVA followed by the Dunnett's post hoc test. $\mathrm{P}<0.05$ was considered to indicate a statistically significant difference.

\section{Results}

Rev reduces cell viability of human gastric cancer cells. To analyze the potential cytotoxic effects of Rev, CCK-8 assay was performed following treatment of GC cell lines AGS and NCI-N87 cells with different concentrations Rev $(0,0.5$, $1,5,10$ and $20 \mu \mathrm{M}$ ) for 24 and $48 \mathrm{~h}$. Rev treatments markedly inhibited cell viability of AGS and NCI-N87 cells in a dose-dependent manner (Fig. 1A), with inhibition becoming significant compared with that in control from $10 \mu \mathrm{M}$ upwards, suggestive of Rev-induced cell death.

Based on these results, the maximum safe drug concentration of Rev of $20 \mu \mathrm{M}$ was selected for subsequent experiments. To verify the Rev-induced reductions of AGS and NCI-N87 cell proliferation, colony formation assay was performed. As shown in Fig. 1B, the number of colonies formed by AGS and NCI-N87 cells stained with crystal violet was significantly decreased following treatment with $\operatorname{Rev}(20 \mu \mathrm{M})$ for $24 \mathrm{~h}$ compared with that in control. These findings suggest that Rev inhibits cell viability and prolifertion in human gastric cancer cells.

Rev inhibits cell migration and invasion of human gastric cancer cells. To explore the effects of Rev on cell migration and invasion in AGS and NCI-N87 cells, wound healing and Transwell assays were conducted. It was found that reductions in the cell free area in the wound observed in those in the control group were significantly attenuated by the presence of Rev in both cell lines (Fig. 2A and B), suggestive of anti-migratory effects mediated by Rev. Using Transwell assay, it was observed that cell invasion was significantly reduced in cells treated with Rev for $48 \mathrm{~h}$ when compared with that in control cells (Fig. 2A and B). In addition, effects of Rev treatment on the expression levels of regulatory proteins of migration were further examined. As shown in Fig. 2C and D, Rev treatment resulted in significant decreases in the levels of MMP-2 and MMP-9 in AGS and NCI-N87 cells. Taken together, these results suggested the anti-migratory and anti-invasive effects of Rev in human gastric cancer cells.

Rev treatment induces apoptosis in human gastric cancer cells. To determine if Rev can induce apoptosis in human GC cells, AGS and NCI-N87 cells were treated with Rev for $24 \mathrm{~h}$ and subsequently used for TUNEL analysis to assess cell apoptosis. The number of GC cells with condensed nuclei (Green) was markedly increased following Rev treatment compared with that in control cells (Fig. 3A). To examine the apoptotic properties of Rev, the expressions of Bax, Bcl-2 and caspase-3/9 were further investigated. Rev treatment resulted in significant increases in the levels of pro-apoptotic proteins Bax, cleaved-caspase-3/9 in AGS and NCI-N87 cells whilst significantly reducing the levels of the anti-apoptotic protein Bcl-2 in response to Rev treatment (Fig. 3B). However, uncleaved caspase-3/9 were not altered in response to Rev treatment. Therefore, these results suggest that $24 \mathrm{~h}$ treatment of gastric cancer cells with Rev can induce cell apoptosis.

Expression of TTK in human gastric cancer cells. To confirm if Rev can target the TTK, TTK expression was detected in 

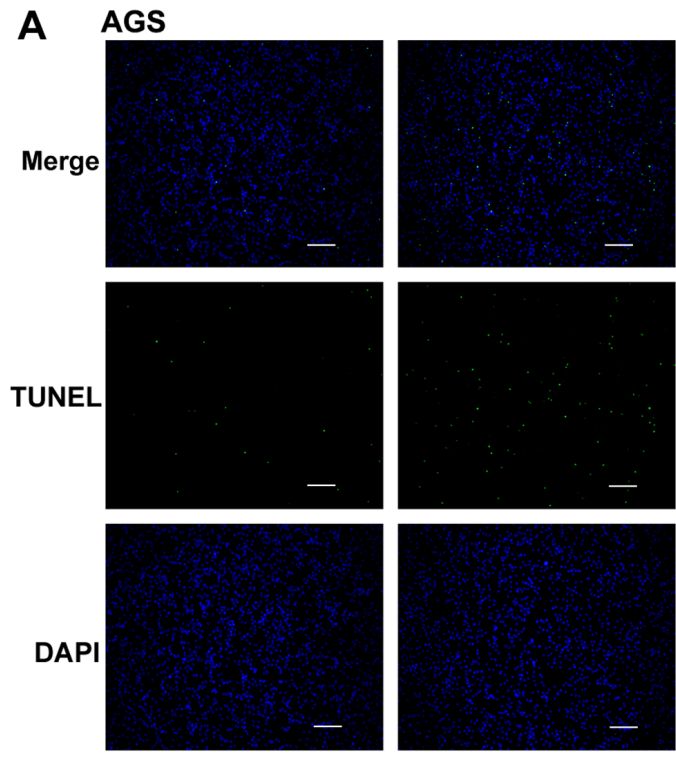

Control

B AGS

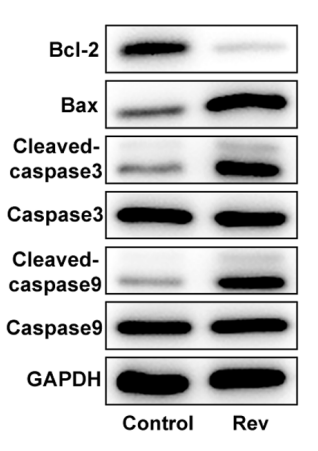

NCI-N87

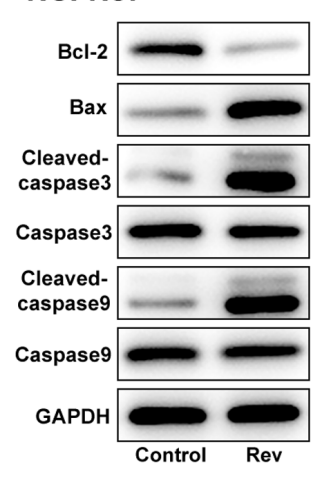

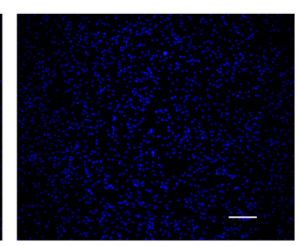

Rev
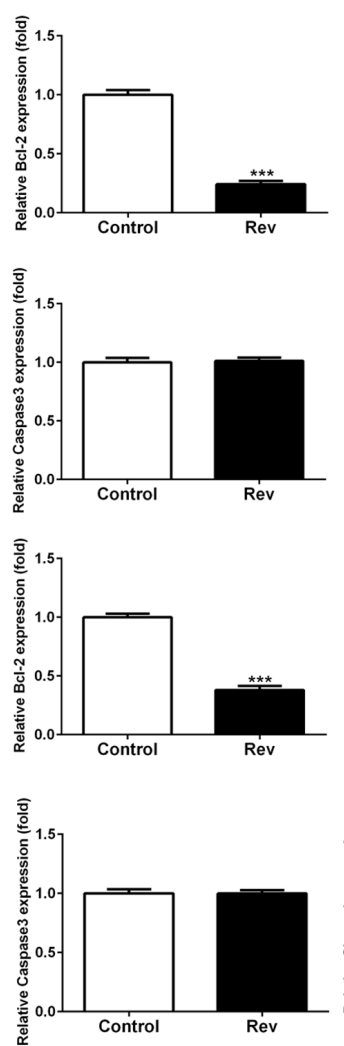

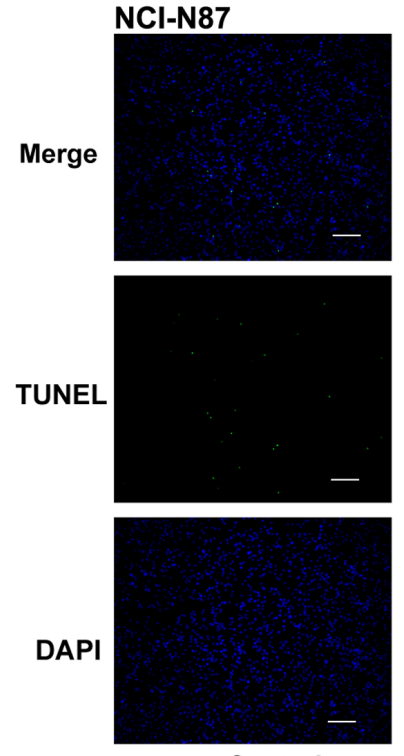

Control
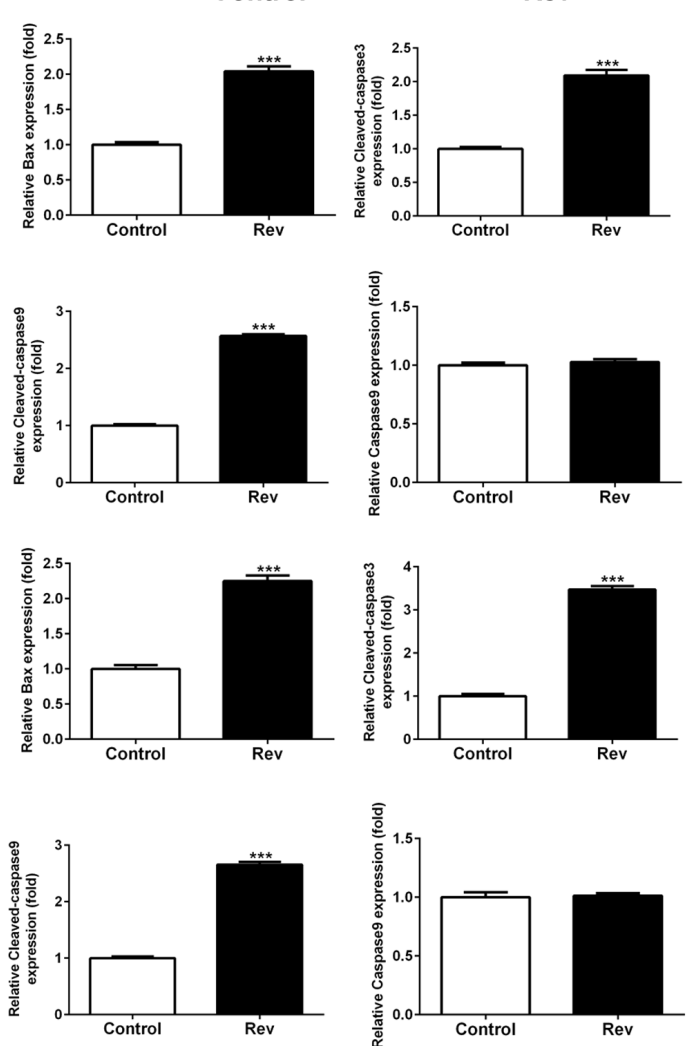
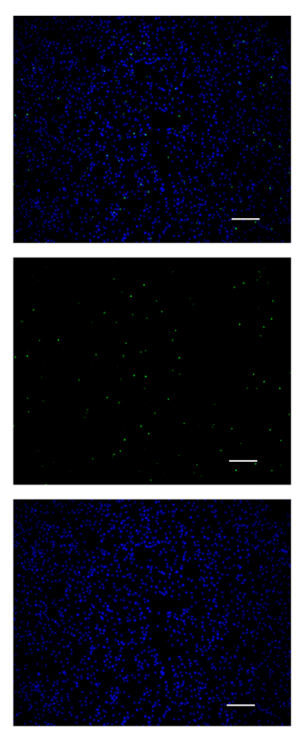

Rev

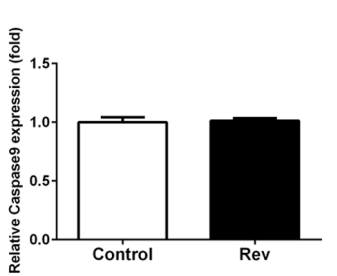

Figure 3. Reversine induces human gastric cancer cell apoptosis. (A) TUNEL staining was performed to observe nuclear morphological alterations under a confocal microscope. Condensed nuclei (green fluorescence), normal nuclei (blue fluorescence), typical of apoptotic cells, were observed in AGS and NCI-N87 cells treated with Rev. Scale bars, $100 \mu \mathrm{m}$. (B) Expression of apoptosis-related markers Bcl-2, Bax, cleaved-caspase3/9 and caspase3/9 were measured by western blotting after Rev treatment in AGS and NCI-N87 cells. ${ }^{* * *} \mathrm{P}<0.001$ vs. Control. Rev, reversine.

GC cells in the presence of Rev using western blotting and RT-qPCR. It was found that TTK expression was significantly higher in both AGS and NCI-N87 cells compared with that GES-1 cells (Fig. 4A). Rev treatment significantly reduced both protein and mRNA TTK expression in both GC cell lines tested (Fig. 4B and C). To explore the effects of TTK further, overexpression plasmids of TTK were used overexpress TTK in AGS and NCI-N87 cells. As shown in Fig. 4D-G, the plasmids of Oe-TTK exhibited good transfection efficiency in both cell lines, which significantly upregulated mRNA and protein levels of TTK compared with those in cells transfected with the Oe-NC plasmid.

Overexpression of TTK attenuates Rev-induced inhibition of cell viability, migration and invasion in human gastric cancer cells. The effect of TTK overexpression on cell viability, migration and invasion following Rev treatment was next examined. Inhibitions of cell viability and colony formation induced by Rev treatment were significantly reversed by Oe-TTK transfection in AGS and NCI-N87 cells 

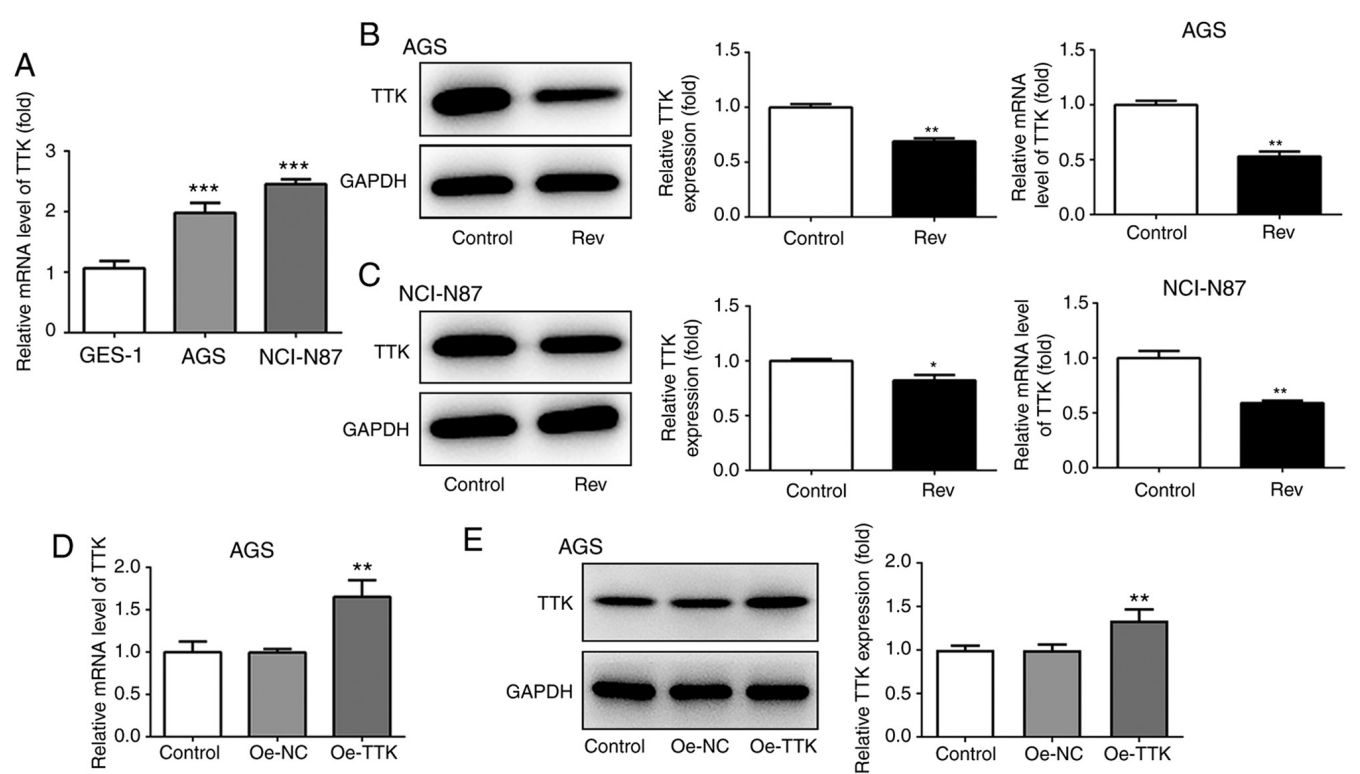

$\mathrm{E}$
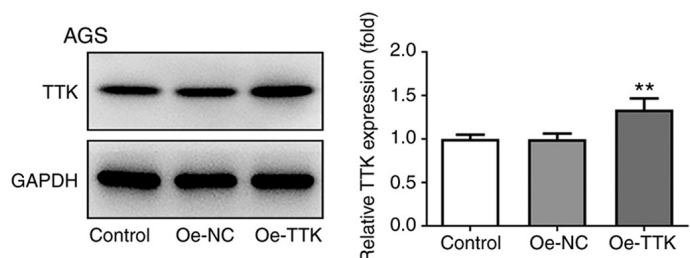

$\mathrm{F}$

G
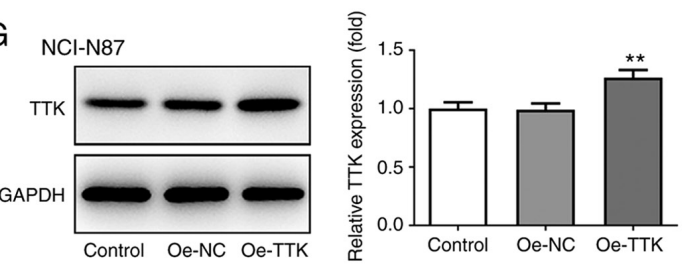

Figure 4. Reversine inhibits TTK expression in human gastric cancer cells. (A) Reverse transcription-quantitative PCR analysis measuring TTK mRNA expression in GC cell lines AGS and NCI-N87, which was compared with that in the human immortalized gastric epithelial cell line GES-1. ${ }^{* * *} \mathrm{P}<0.001$ vs. GES-1. mRNA and protein expression of TTK was evaluated after Rev treatment in (B) AGS and (C) NCI-N87 cells. ** P $<0.01$ vs. Control. Following TTK-specific overexpression, TTK (D) mRNA and (E) protein expression levels were measured in AGS cells. Following TTK-specific overexpression, TTK (D) mRNA and (E) protein expression levels were measured in NCI-N87 cells. TTK protein expression levels were measured in (F) AGS and (G) NCI-N87 cells following TTK-specific overexpression. ${ }^{* *} \mathrm{P}<0.01$ vs. control or Oe-NC. Rev, reversine; $\mathrm{NC}$, negative control; Oe, overexpression.

A

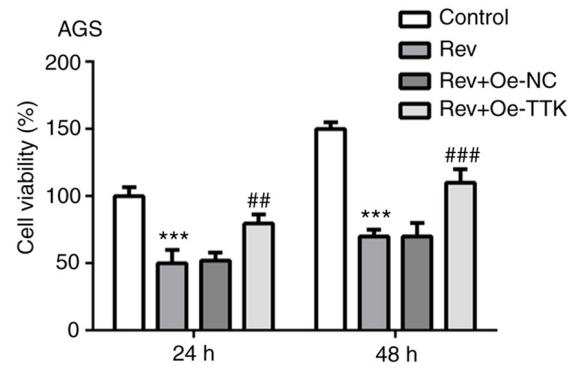

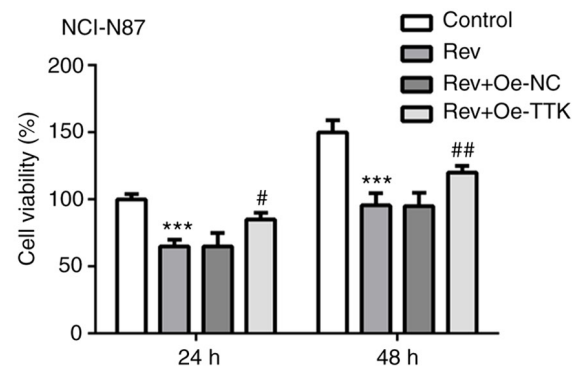

B AGS
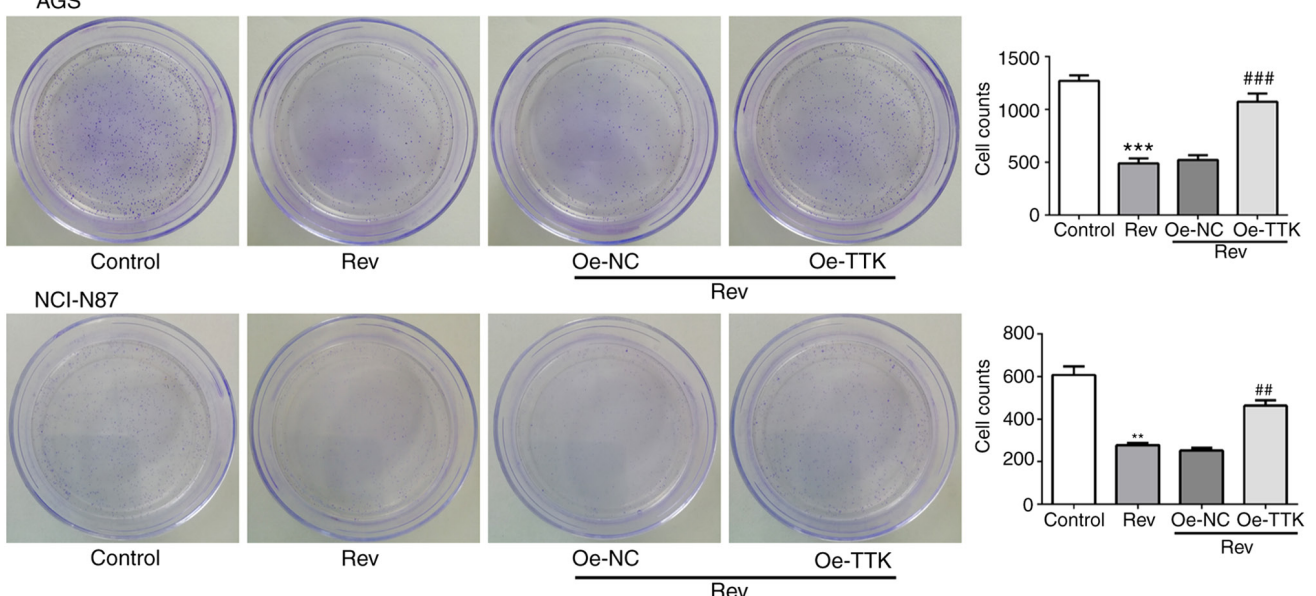

Figure 5. TTK overexpression attenuates reversine-induced inhibition of cell growth in human gastric cancer cells. (A) CCK-8 assay was performed to measure cell viability of AGS and NCI-N87 cells. (B) Representative images of cells in colony formation assay are shown in left and quantitative data are presented in the right panel. ${ }^{* *} \mathrm{P}<0.01$ and ${ }^{* * *} \mathrm{P}<0.001$ vs. Control; ${ }^{\#} \mathrm{P}<0.05,{ }^{\# \#} \mathrm{P}<0.01$ and ${ }^{\# \# \#} \mathrm{P}<0.001$ vs. Rev. Rev, reversine; NC, negative control; Oe, overexpression. 


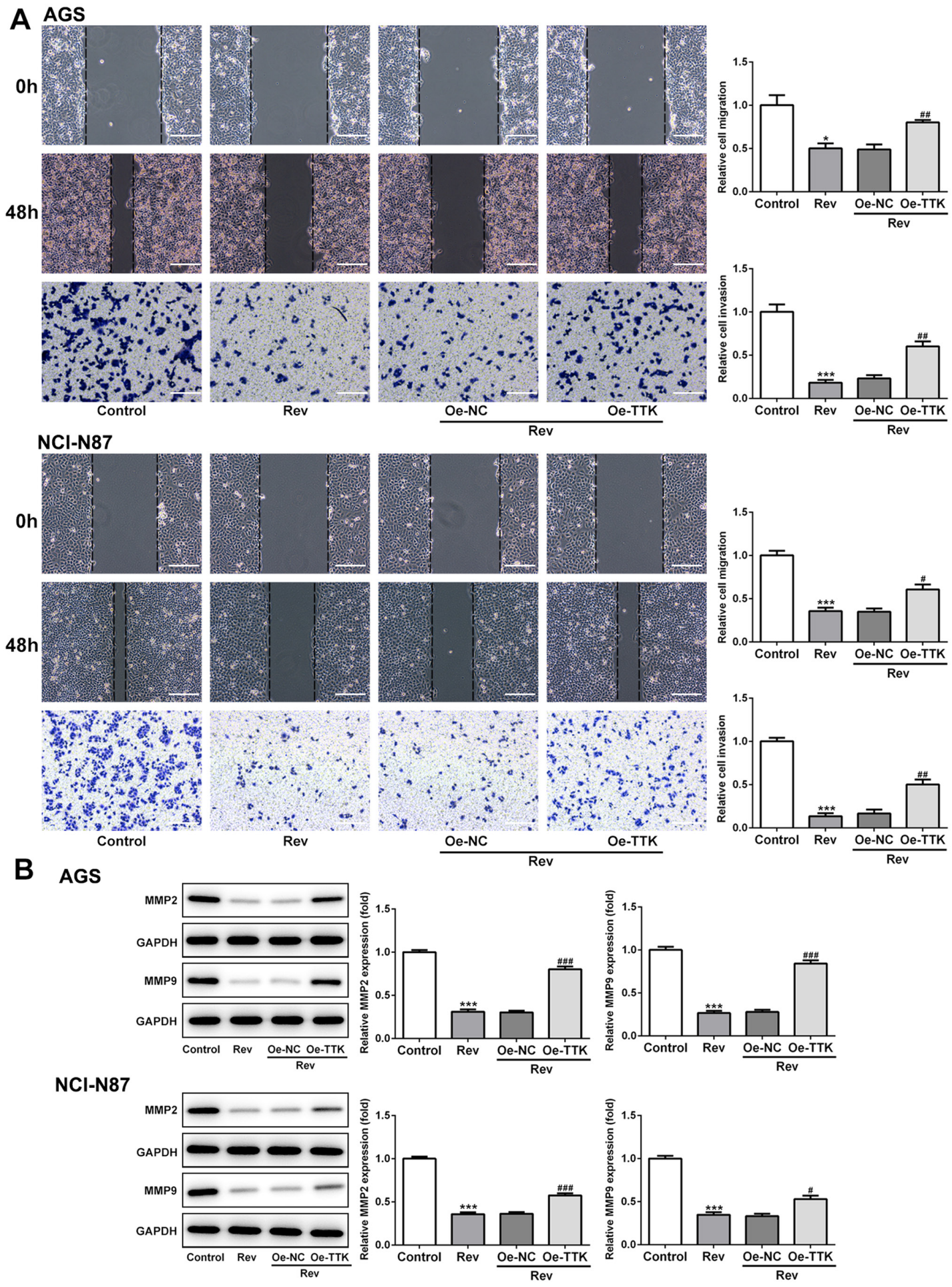

Figure 6. TTK overexpression attenuates reversine-induced inhibition of cell migration and invasion in human gastric cancer cells. (A) Wound healing and Transwell assays were performed to measure the migration ability of AGS and NCI-N87 cells transfected with the TTK overexpression vector after Rev treatment. Scale bars, $40 \mu \mathrm{m}$. (B) Western blot analysis was used to measure changes in the protein expression of migration-related markers MMP2 and MMP9. "P<0.05 and ${ }^{* * * *} \mathrm{P}<0.001$ vs. Control; ${ }^{\#} \mathrm{P}<0.05,{ }^{\# \#} \mathrm{P}<0.01$ and ${ }^{\# \# \#} \mathrm{P}<0.001$ vs Rev. Rev, reversine; MMP, matrix metalloproteinase; NC, negative control; Oe, overexpression.

(Fig. 5A and B). In addition, overexpression of TTK significantly reversed the inhibitory effects of Rev on the migratory and invasive capacities of GC cells (Fig. 6A). Expression levels of MMP2 and MMP9 were also significantly elevated in Rev-treated GC cells transfected with Oe-TTK compared with those in cells treated with Rev alone (Fig. 6B). These results suggest that the Rev-induced inhibition of GC cell migration and invasion is mediated by suppressing TTK expression.
Overexpression of TTK attenuates reversine-induced induction of apoptosis in human gastric cancer cells. To determine if Rev can induce apoptosis in GC cells by targeting TTK, TUNEL and western blotting were performed to assess cell apoptosis and expression of apoptosis-related proteins. Increases in the number of apoptotic cells, which were stained green by TUNEL, following Rev treatment was markedly attenuated in AGS and NCI-N87 cells by Oe-TTK transfection 


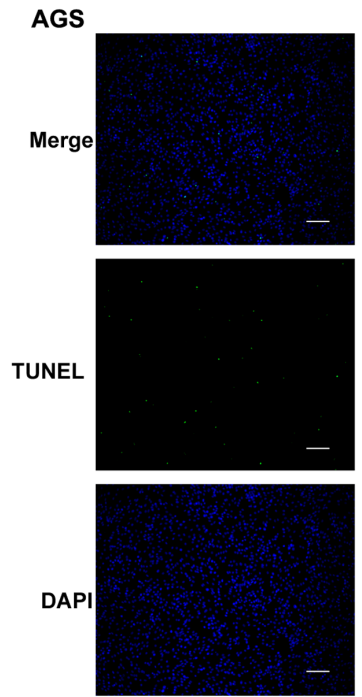

Control
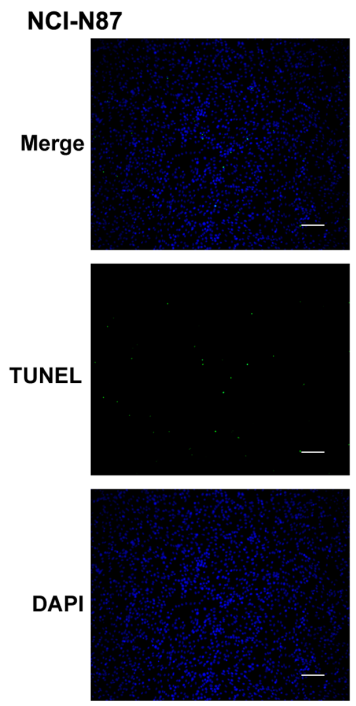

Control
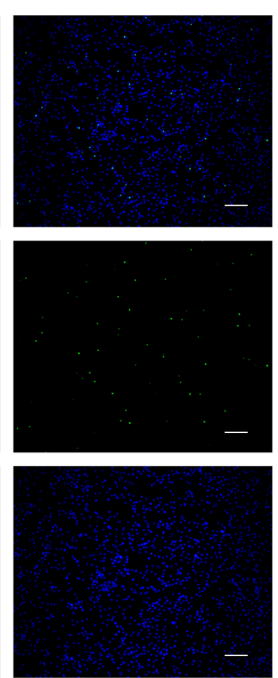

$\operatorname{Rev}$
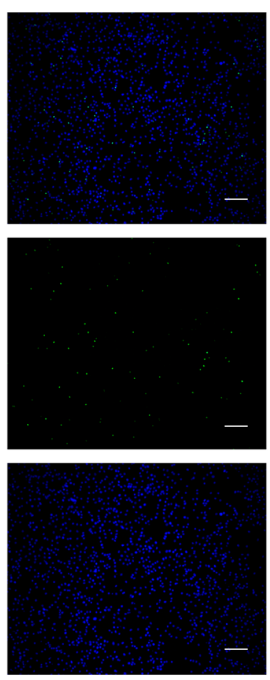

Rev
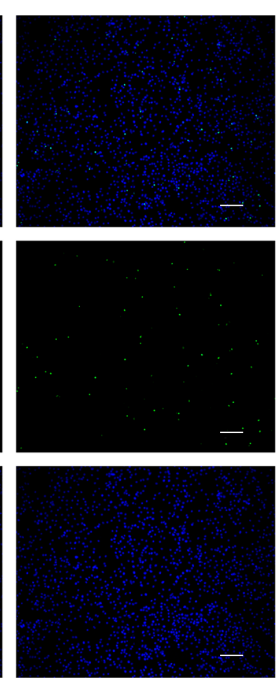

Oe-NC
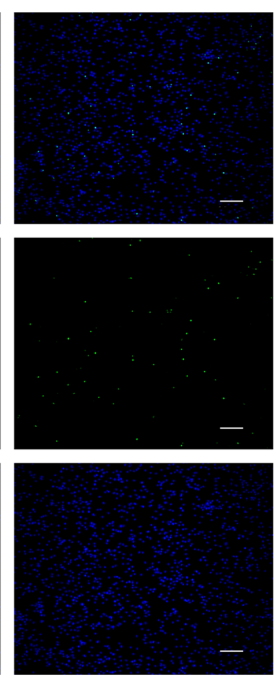

Oe-NC
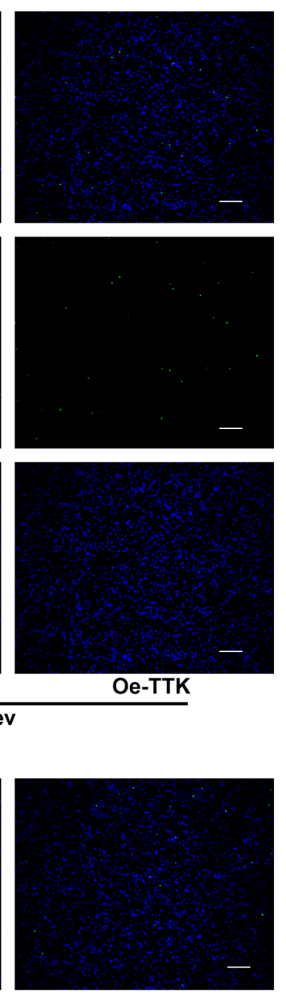

Rev
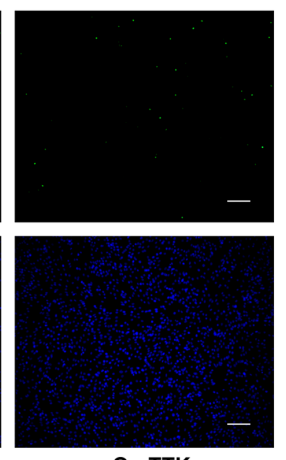

Oe-TTK

Figure 7. TTK overexpression attenuates reversine-induced induction of apoptosis in human gastric cancer cells. Apoptosis of AGS and NCI-N87 cells was analyzed by TUNEL staining after TTK overexpression. Representative fluorescence images were shown. Condensed nuclei (green fluorescence), normal nuclei (blue fluorescence). Scale bars, $100 \mu \mathrm{m}$. Rev, reversine; NC, negative control; Oe, overexpression.

(Fig. 7). Furthermore, TTK overexpression significantly abrogated the Rev-induced elevations in cleaved-caspase-3 and -9 activation and Bcl-2 inhibition. However, TTK overexpression did not significantly affect uncleaved caspase- 3 and -9 protein expression (Fig. 8). Therefore, these observations suggest that Rev treatment promotes apoptosis by directly inhibiting TTK in human gastric cancer cells.

\section{Discussion}

Rev is a synthetic purine analogue that has been previously reported to induce the de-differentiation of the murine myoblast cell line into multipotent progenitor cells, which can subsequently re-differentiate into other different cell types (4). Rev increased the plasticity of bone marrow-derived mesenchymal stem cells for the generation of cardiomyocytes in vitro (19). In addition, Rev induced myoblast redifferentiation into cell types of neural and mesodermal lineages (20). Rev also exerted an inhibitive effect on human renal carcinoma cells via induction of cell apoptosis and polyploidy (21). A number of studies have suggested that Rev confers tumor-suppressive effects against several cancer cells types, including colorectal and breast cancer $(5,7,9)$. However, studies evaluating the effects of Rev on human GCs has not been previously reported. Therefore, the impact of Rev on tumor cell behavior and its association with TTK expression in human gastric cancer cells were investigated in the present study, which found Rev to be a potential anti-GC agent.

Dysregulation of cell growth and apoptosis are important for cancer development and progression (22). In the present study, Rev notably reduced the cell viability of the two GC cell lines tested in a concentration-dependent manner, consistent with previous findings that Rev exerted anticancer effects by suppressing CRC cell growth (9). Furthermore, Rev treatment significantly reduced colony formation. TUNEL staining was performed and nuclear condensation was also evaluated in the 

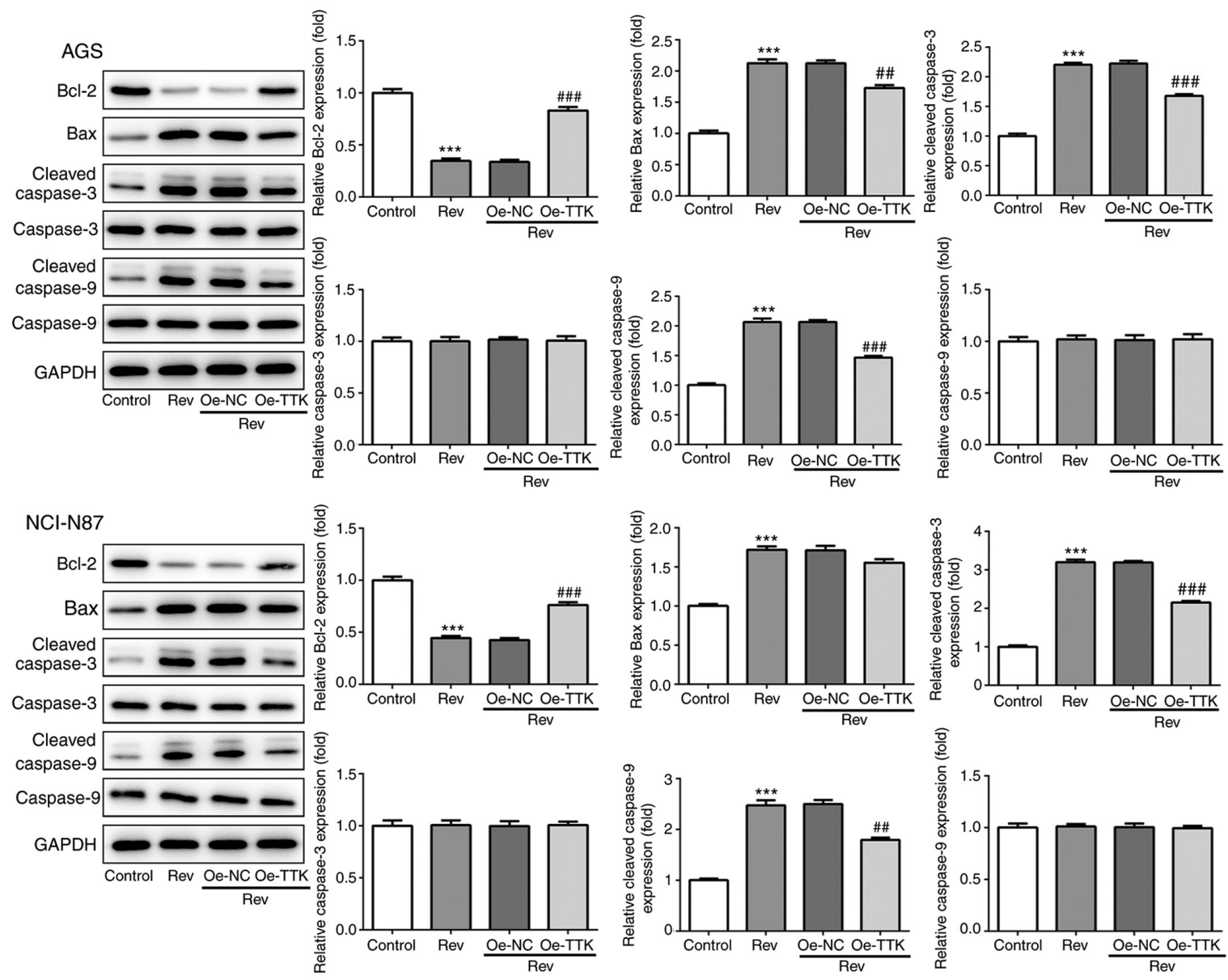

Figure 8. TTK overexpression attenuates reversine-induced induction of apoptosis in human gastric cancer cells. Expression of apoptosis-related markers Bcl-2, Bax, cleaved-caspase $3 / 9$ and caspase $3 / 9$ were measured by western blotting after TTK overexpression in AGS and NCI-N87 cells. Representative images of western blotting are presented on the left and quantified on the right. ${ }^{* * *} \mathrm{P}<0.001$ vs. control; ${ }^{\# \#} \mathrm{P}<0.01$ and ${ }^{\# \# t} \mathrm{P}<0.001 \mathrm{vs}$. Rev. Rev, reversine; $\mathrm{NC}$, negative control; Oe, overexpression.

present study. To verify he effects of Rev on the apoptosis of GC cells, expression of the anti-apoptotic protein Bcl-2, the pro-apoptotic protein Bax and the central component of the apoptotic cascade caspase-3/9, were measured. Rev treatment induced apoptosis by activating Bax and caspase-3/9 whilst suppressing the expression of Bcl-2. These results suggest that Rev exerts cytotoxicity on GC cells and has potential applications for anticancer treatment.

Metastasis is a major cause of chemotherapeutic failure and cancer mortality, such that gastric cancer is one of the most invasive and metastatic type of cancer (2). Recently, Rev was demonstrated to suppress the migration and invasion of human CRC (9). In the present study, GC cells were exposed to Rev for $24 \mathrm{~h}$, where it was found that Rev significantly suppressed the migration and invasion of GC cells using wound healing and Transwell assays. The potential anti-metastatic effects of Rev was in line with previous findings that Rev functions as a potent anti-migratory agent in invasive cancer cells $(8,23)$. MMPs serve important roles in many physiological and pathological processes, including angiogenesis, tumor invasion and metastasis (24). Lyu et al (25) found that Marimastat, an MMP inhibitor, could inhibit MMP2 and MMP 9, thereby inhibiting tumor growth, invasion and metastasis in breast cancer cells. In the present study, Rev reduced the expression of MMP2 and MMP9 in GC cells. This suggest that Rev can potentially inhibit GC cancer cell metastasis, consistent with a previous finding (8).

A previous study performed in vitro showed that TTK knockdown inhibited the proliferation, invasion and migration of prostate cancer cells whilst promoting cell apoptosis (26). In addition, in vivo experiments showed that TTK gene knockout inhibited tumorigenesis in mice injected with prostate cancer cells (26). Hudler et al (16) confirmed that polymorphisms in the gene of the mitotic kinase TTK could have an effect on the risk of gastric tumorigenesis and adenocarcinoma development. In the present study, it was found that TTK was overexpressed in GC cells compared with that in the normal human gastric epithelial cell line, which was in turn reduced by Rev treatment. Therefore, it could be hypothesized that TTK can serve an important role in GC as a downstream target of Rev. Therefore, overexpression of TTK was subsequently used to assess the association between Rev and TTK expression in GC cells. The present study demonstrated that TTK overexpression reversed the Rev-induced reductions in cell viability, colony formation, migration, invasion and Rev-induced apoptosis in GC cells, consistent with a previous study, which also reported that TTK can inhibit the proliferation of prostate cancer (26). However, further experimentation is required to explore the specific underlying mechanism. Therefore, TTK appears to be an attractive target for the development of novel therapeutics against 
gastric cancer. In the present study, the effects and potential mechanism of Rev on GC was only tested on cell lines without further verification by in vivo experiments. The in vivo role of Rev in GC would need to be explored in further studies.

Overall, the present study provided evidence that Rev treatment suppressed GC physiology by inhibiting of cell viability, migration and invasion, whilst inducing apoptosis, by downregulating TTK expression in human GC cells. Results from the present study suggest that Rev may be used as a novel anticancer agent for human GC, such that TTK may serve as an attractive target for cancer therapy.

\section{Acknowledgements}

Not applicable.

\section{Funding}

No funding was received.

\section{Availability of data and materials}

All data generated or analyzed during this study are included in this published article.

\section{Authors' contributions}

PX and ZJ designed the experiments. PX, JL and DJ performed the experiments and analyzed the data. JL and DJ authenticated the raw data. All the authors read and approved the final version of this manuscript.

\section{Ethics approval and consent to participate}

Not applicable.

\section{Patient consent for publication}

Not applicable.

\section{Competing interests}

The authors declare that they have no competing interests.

\section{References}

1. Correa P: Gastric cancer: Overview. Gastroenterol Clin North Am 42: 211-217, 2013

2. Song Z, Wu Y, Yang J, Yang D and Fang X: Progress in the treatment of advanced gastric cancer. Tumour Biol: Jul 3, 2017 (Epub ahead of print). doi: 10.1177/1010428317714626.

3. Wu H, Wang W, Tong S and Wu C: Nucleostemin regulates proliferation and migration of gastric cancer and correlates with its malignancy. Int J Clin Exp Med 8: 17634-17643, 2015.

4. Chen S, Zhang Q, Wu X, Schultz PG and Ding S: Dedifferentiation of lineage-committed cells by a small molecule. J Am Chem Soc 126: 410-411, 2004

5. Hsieh TC, Traganos F, Darzynkiewicz Z and Wu JM: The 2,6-disubstituted purine reversine induces growth arrest and polyploidy in human cancer cells. Int J Oncol 31: 1293-1300, 2007.

6. Lu YC, Lee YR, Liao JD, Lin CY, Chen YY, Chen PT and Tseng YS: Reversine induced multinucleated cells, cell apoptosis and autophagy in human non-small cell lung cancer cells. PLoS One 11: e0158587, 2016.
7. Kuo CH, Lu YC, Tseng YS, Shi CS, Chen SH, Chen PT, Wu FL, Chang YP and Lee YR: Reversine induces cell cycle arrest, polyploidy, and apoptosis in human breast cancer cells. Breast Cancer 21: 358-369, 2014

8. Jemaa M, Abassi Y, Kifagi C, Fezai M, Daams R, Lang F and Massoumi R: Reversine inhibits colon carcinoma cell migration by targeting JNK1. Sci Rep 8: 11821, 2018.

9. Park YL, Ha SY, Park SY, Choi JH, Jung MW, Myung DS, Kim HS and Joo YE: Reversine induces cell cycle arrest and apoptosis via upregulation of the Fas and DR5 signaling pathways in human colorectal cancer cells. Int J Oncol 54: 1875-1883, 2019.

10. Liu X, Liao W, Yuan Q, Ou Y, and Huang J: TTK activates Akt and promotes proliferation and migration of hepatocellular carcinoma cells. Oncotarget 6: 34309-34320, 2015.

11. Kaistha BP, Honstein T, Muller V, Bielak S, Sauer M, Kreider R, Fassan M, Scarpa A, Schmees C, Volkmer H, et al: Key role of dual specificity kinase TTK in proliferation and survival of pancreatic cancer cells. Br J Cancer 111: 1780-1787, 2014.

12. King JL, Zhang B, Li Y, Li KP, Ni JJ, Saavedra HI and Dong JT: TTK promotes mesenchymal signaling via multiple mechanisms in triple negative breast cancer. Oncogenesis 7: 69, 2018.

13. Wang J, Xie Y, Bai X, Wang N, Yu H, Deng Z, Lian M, Yu S, Liu H, Xie W and Wang M: Targeting dual specificity protein kinase TTK attenuates tumorigenesis of glioblastoma. Oncotarget 9: 3081-3088, 2018.

14. Ahn CH, Kim YR, Kim SS, Yoo NJ and Lee SH: Mutational analysis of TTK gene in gastric and colorectal cancers with microsatellite instability. Cancer Res Treat 41: 224-228, 2009.

15. Finetti P, Cervera N, Charafe-Jauffret E, Chabannon C, Charpin C, Chaffanet M, Jacquemier J, Viens P, Birnbaum D and Bertucci F: Sixteen-kinase gene expression identifies luminal breast cancers with poor prognosis. Cancer Res 68: 767-776, 2008.

16. Hudler P, Britovsek NK, Grazio SF and Komel R: Association between polymorphisms in segregation genes BUB1B and TTK and gastric cancer risk. Radiol Oncol 50: 297-307, 2016.

17. Wang YW, Zhu ZG, Liu BY, Gu QL, Li JF, Qu Y, Chen XH and Lin YZ: Expression and clinical significance of cancer-related gene MPS-1 in gastric cancer. Zhonghua Wei Chang Wai Ke Za Zhi 8: 503-506, 2005 (In Chinese).

18. Livak KJ and Schmittgen TD: Analysis of relative gene expression data using real-time quantitative PCR and the 2(-Delta Delta C(T)) method. Methods 25: 402-408, 2001.

19. Pikir BS, Susilowati H, Hendrianto E and Abdulrantam F: Reversin increase the plasticity of bone marrow-derived mesenchymal stem cell for generation of cardiomyocyte in vitro. Acta Med Indones 44: 23-27, 2012.

20. Lee EK, Bae GU, You JS, Lee JC, Jeon YJ, Park JW, Park JH, Ahn SH, Kim YK, Choi WS, et al: Reversine increases the plasticity of lineage-committed cells toward neuroectodermal lineage. J Biol Chem 284: 2891-2901, 2009.

21. Cheng L, Wang H, Guo K, Wang Z, Zhang Z, Shen C, Chen L and Lin J: Reversine, a substituted purine, exerts an inhibitive effect on human renal carcinoma cells via induction of cell apoptosis and polyploidy. Onco Targets Ther 11: 1025-1035, 2018.

22. Llambi F and Green DR: Apoptosis and oncogenesis: Give and take in the BCL-2 family. Curr Opin Genet Dev 21: 12-20, 2011.

23. Jemaà M, Galluzzi L, Kepp O, Boileve A, Lissa D, Senovilla L, Harper F, Pierron G, Berardinelli F, Antoccia A, et al: Preferential killing of p53-deficient cancer cells by reversine. Cell Cycle 11: 2149-2158, 2012

24. Huang H, Wu K, Ma J, Du Y, Cao C and Nie Y: Dopamine D2 receptor suppresses gastric cancer cell invasion and migration via inhibition of EGFR/AKT/MMP-13 pathway. Int Immunopharmacol 39: 113-120, 2016.

25. Lyu Y, Xiao Q, Yin L, Yang L and He W: Potent delivery of an MMP inhibitor to the tumor microenvironment with thermosensitive liposomes for the suppression of metastasis and angiogenesis. Signal Transduct Target Ther 4: 26, 2019.

26. Chen S, Wang J, Wang L, Peng H, Xiao L, Li C, Lin D and Yang K: Silencing TTK expression inhibits the proliferation and progression of prostate cancer. Exp Cell Res 385: 111669, 2019.

This work is licensed under a Creative Commons Attribution-NonCommercial-NoDerivatives 4.0 International (CC BY-NC-ND 4.0) License. 